Received: 2016.11.17

Accepted: 2017.01 .14

Published: 2017.09.15

\section{Role of Diffusion-Weighted Magnetic Resonance (MR) Imaging in Differentiation Between Graves' Disease and Painless Thyroiditis}

Authors' Contribution:
A Study Design
B Data Collection
C Statistical Analysis
D Data Interpretation
E Manuscript Preparation
F Literature Search
G Funds Collection

\author{
Ahmed Abdel Khalek Abdel Razek ${ }^{1 \mathrm{ABCDEF}}$, Sieza Samir Abd Allah ${ }^{1 \mathrm{BCDE}}$, \\ Amr Abd El-hamid El-Said ${ }^{2 B C D F}$ \\ 1 Department of Diagnostic Radiology, Mansoura Faculty of Medicine, Mansoura, Egypt \\ 2 Department of Internal Medicine, Mansoura Faculty of Medicine, Mansoura, Egypt
}

Author's address: Sieza Samir Abd Allah, Department of Diagnostic Radiology, Mansoura Faculty of Medicine, Mansoura, Egypt, e-mail: sieza_s@mans.edu.eg

(2)

Background: $\quad$ To assess the role of diffusion-weighted MR imaging in differentiation between Graves' disease and painless thyroiditis.

Material/Methods:

Results: $\quad$ There was a significant difference in the ADC value of the thyroid gland between patients and the control group $(P=0.001)$. The mean $A D C$ value of the thyroid gland in Graves' disease was $2.03 \pm 0.28 \times 10^{-3} \mathrm{~mm}^{2} / \mathrm{sec}$, and in patients with painless thyroiditis $1.46 \pm 0.22 \times 10^{-3} \mathrm{~mm}^{2} / \mathrm{sec}_{\text {, }}$ respectively. There was a significant difference in the ADC values between Graves' disease and painless thyroiditis $(P=0.001)$. When the ADC value of $1.45 \times 10^{-3} \mathrm{~mm}^{2} / \mathrm{sec}$ was used as a threshold value for differentiating Graves' disease from painless thyroiditis, the best result was obtained with area under the curve of 0.934 , accuracy of $83.8 \%$, sensitivity of $95.8 \%$, and specificity of $61.5 \%$. The mean $\mathrm{ADC}$ value of the thyroid gland in patients positively correlated with serum TRAb and Tc-99m uptake ( $r=0.57, P=0.001$ and $r=0.74, P=0.001$, respectively).

Conclusions: We concluded that ADC values of the thyroid gland can be used to differentiate Graves' disease from painless thyroiditis in patients with untreated thyrotoxicosis.

MeSH Keywords: $\quad$ Diffusion Magnetic Resonance Imaging • Goiter • Thyroiditis

PDF file: http://www.polradiol.com/abstract/index/idArt/902416

\section{Background}

Thyrotoxicosis results from thyroid hormone overproduction in Graves' disease, or from thyroid hormone leakage from damaged or inflamed thyroid tissue in painless thyroiditis. Differentiation between these two entities is important for selection of proper therapy. Graves' disease is treated with anti-thyroid drugs, radioactive iodine, and thyroidectomy, whereas destruction-induced thyrotoxicosis in painless thyroiditis is a self-limiting disorder that can be managed conservatively. If painless thyroiditis is misdiagnosed as Graves' disease and treated with antithyroid drugs, hypothyroidism, drug-induced hepatitis, and leucopenia may occur [1-5]. Graves' disease is an autoimmune disorder characterized by the formation of autoantibodies that are directed against the thyroid stimulating hormone receptors. These antibodies lead to thyroid follicular hyperplasia with a subsequent excessive thyroid hormone production and suppression of thyroid stimulating hormone. Painless thyroiditis, also known as silent thyroiditis or subacute lymphocytic thyroiditis, is caused by lymphocytic infiltration of the thyroid follicles, secondary 
to an autoimmune response. This lymphocytic infiltration leads to thyroid follicular disruption with a release of an excess of thyroid hormones and subsequent transient thyrotoxicosis [1-3]. The presence of serum anti-thyrotropin receptor antibodies (TRAb) is regarded as a useful marker that can discriminate between Graves' disease and painless thyroiditis. However, it is not widely available with overlap in its values [6-9]. Different imaging modalities are used for assessing patients with thyrotoxicosis [5]. Color Doppler ultrasonography was used to investigate the pathogenesis of thyrotoxicosis. However, this examination is not satisfactory in all patients, and the results are operator-dependent [10-14]. Scintigraphy of the thyroid gland is the gold standard for evaluation of thyrotoxicosis, but it is associated with radiation exposure, long examination time, and is contraindicated during lactation [15-17].

Diffusion-weighted MR imaging is a noninvasive imaging tool with no exposure to ionizing radiation. Diffusionweighted imaging provides information on the random motion of water molecules in tissues, which indicates tissue cellularity [18-20]. Diffusion-weighted MR imaging was used for characterization of thyroid nodules [21-23], evaluation of diffuse thyroid diseases [24], assessment of Graves' disease activity, and prediction of therapeutic outcomes [25]. Two recent studies discussed the role of diffusion-weighted MR imaging in differentiating between Graves' disease, Hashimoto's thyroiditis, and painless thyroiditis [26,27].

The aim of this work was to assess the role of diffusionweighted MR imaging in the differentiation between Graves' disease and painless thyroiditis.

\section{Material and Methods}

\section{Patients}

Approval of the institutional review board for this study was obtained, and informed consents were obtained from patients and volunteers. This prospective study was conducted in 42 consecutive patients with untreated thyrotoxicosis. Their age ranged from 22 to 52 years, the mean age was 44 years. Five patients were excluded from the study due to presence of multiple thyroid nodules (3 patients) and claustrophobia (2 patients). A control group consisting of 15 volunteers was randomly selected from patients who were referred for cervical MR imaging examinations. The mean age and male-to-female ratio of the two groups were matched. All patients and controls underwent diffusionweighted MR imaging of the thyroid gland and laboratory testing; only the patients underwent thyroid scintigraphy. Graves' disease was diagnosed on the basis of clinical findings and laboratory tests that showed high values of free thyroxine (FT4) and free tri-iodothyronine (FT3), low levels of thyrotropin-stimulating hormone (TSH), and increased TSH receptor antibody (TRAb) titer. Painless thyroiditis was diagnosed based on increased levels of FT4 and FT3 for less than 3 months, and/or later development of transient hypothyroidism, without neck pain or tenderness. This study involved 24 patients with Graves' disease (female/male; 17/7, mean age 43.3 years) and 13 patients with painless thyroiditis (female/male; 8/5, mean age 41.2 years).

\section{MR imaging}

MR images were obtained with a superconducting $1.5 \mathrm{~T}$ MR imaging unit (Achieva, Philips Healthcare, Best, The Netherlands). We used a dedicated eight-channel neck coil (Achieva Sense; Philips Healthcare, Best, Netherlands). Patients were placed in a supine position, with the CP-neck array coil placed in such a way that the thyroid gland was located in the central portion of the field of view. Axial, fast filed echo (FFE) images were acquired using the following parameters: TR/TE $=800 / 20 \mathrm{msec}$, flip angle $=20$ degrees, section thickness $=4 \mathrm{~mm}$, intersection gap $=1 \mathrm{~mm}$, matrix $=192 \times 256$, and field of view $(\mathrm{FOV})=20-23 \mathrm{~cm}$. Diffusion-weighted $\mathrm{MR}$ imaging of the thyroid gland was performed in axial plane using the spin-echo type of echo-planar sequence. The imaging parameters were $\mathrm{TR} / \mathrm{TE}=4200 / 140 \mathrm{msec}, \mathrm{NEX}=6$, bandwidth $=1345 \mathrm{~Hz} /$ pixel, field of view $=20-23 \mathrm{~cm}$, matrix $=256 \times 128$, slice thickness $=4 \mathrm{~mm}$, intersection gap $=1 \mathrm{~mm}$ and $b$-values $=0,400$ and $800 \mathrm{sec} / \mathrm{mm}^{2}$. The diffusion gradients were applied in three orthogonal directions ( $\mathrm{X}, \mathrm{Y}$, and $\mathrm{Z}$ ). The scanning time of diffusion-weighted MR imaging was 135 seconds. The ADC maps were automatically generated on the operating console with commercially available software.

\section{Image analysis}

Image analysis was performed in consensus by two radiologists (AA and SS), experts in head and neck imaging (20 and 10 years of experience). A region of interest was drawn around both thyroid lobes and isthmus in 3 different sections of the ADC map (Figure 1). The sections that were selected for analysis showed homogeneous thyroid parenchyma that was not affected by artifacts due to chemical shift, magnetic susceptibility, or vascular motion. The $\mathrm{ADC}$ value was automatically calculated and expressed in $10^{-3} \mathrm{~mm}^{2} / \mathrm{sec}$. The final ADC per-subject value that was used for statistical analysis was the average of these three $\mathrm{ADC}$ values. In the control group, the sections that showed homogeneous thyroid parenchyma were similarly selected for analysis.

\section{Thyroid scintigraphy}

After an intravenous injection of $185 \mathrm{MBq}$ Tc-99m pertechnetate $(5 \mathrm{mCi})$, thyroid scans were performed using a gamma camera with low-energy, high-sensitivity, parallelhole collimator. The collimator was centered over the thyroid at a distance of $10 \mathrm{~cm}$. Imaging commenced approximately 20 minutes after injection. The images were recorded for $180 \mathrm{~s}$, with a matrix size of $512 \times 512$ and a 2 -fold magnification. A region of interest was drawn around the borders of the thyroid gland, and another region of interest in the supraclavicular area was used for background subtraction to calculate the thyroid uptake [16].

\section{Laboratory tests}

Serum concentrations of TSH, FT4, and FT3 were measured with an electrochemiluminescence immunoassay (ECLUSYS TSH, FT 4 and FT 3; Roche Diagnostics KK, Tokyo, Japan). Serum TRAb levels were measured by a 


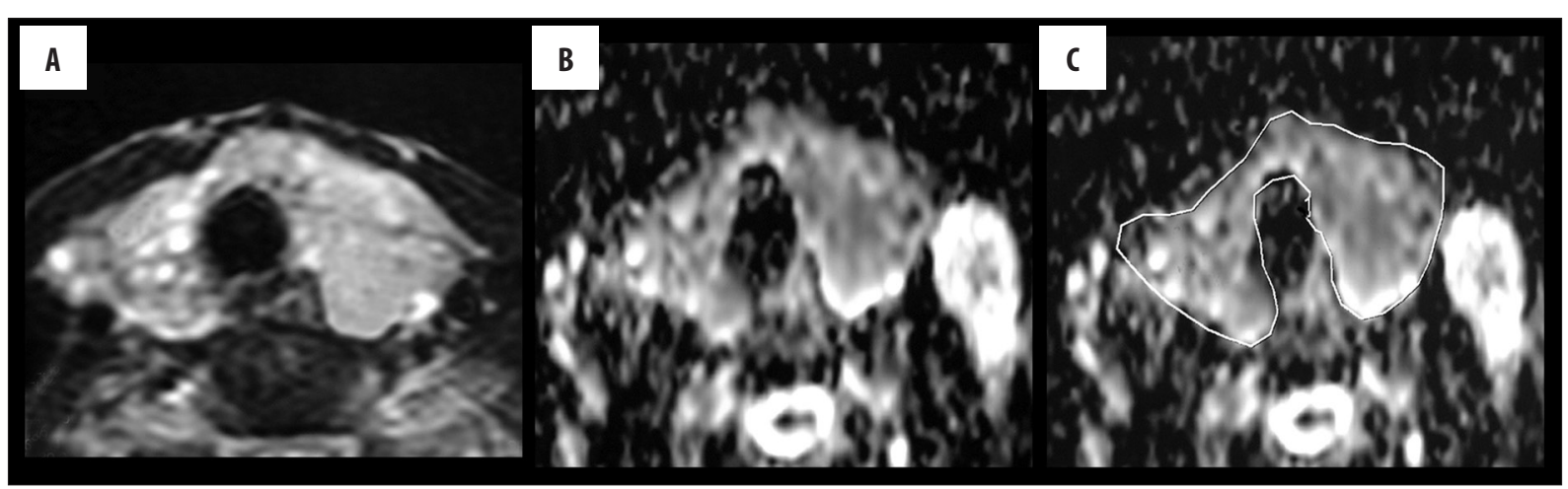

Figure 1. Graves' disease: (A) Axial, fast filed echo image shows diffusely enlarged both thyroid lobes and isthmus. (B) ADC map shows high ADC values of the thyroid gland $\left(1.9 \times 10^{-3} \mathrm{~mm}^{2} / \mathrm{sec}\right)$. (C) $A D C$ map with localization of the region of interest.

Table 1. The mean, standard deviation, minimum, and maximum ADC values $\left(\times 10^{-3} \mathrm{~mm}^{2} / \mathrm{sec}\right)$ of the thyroid gland in Graves' disease, painless thyroiditis, thyrotoxicosis, and in control group.

\begin{tabular}{lccc}
\hline & Mean \pm SD & Minimum & Maximum \\
\hline Graves' disease $(\mathrm{n}=24)$ & $2.03 \pm 0.28$ & 1.45 & 2.5 \\
\hline Painless thyroiditis $(\mathrm{n}=13)$ & $1.46 \pm 0.22$ & 1.22 & 1.91 \\
\hline Thyrotoxicosis $(\mathrm{n}=37)$ & $1.83 \pm 0.38$ & 1.22 & 2.5 \\
\hline Control group $(\mathrm{n}=15)$ & $1.02 \pm 0.14$ & 0.85 & 1.3 \\
\hline
\end{tabular}

Table 2. The median, minimum, and maximum of Tc-99m uptake values and the thyroid function tests in patients with Graves' disease, painless thyroiditis, thyrotoxicosis, and control group.

\begin{tabular}{lccccc}
\hline & Tc-99m uptake \% & TRAb $(\mathbf{I U} / \mathbf{L})$ & TSH $(\boldsymbol{\mu l} \mathbf{l} / \mathbf{m l})$ & FT3 $\mathbf{p g} / \mathbf{m l})$ & FT4 $(\mathbf{p g} / \mathbf{d l})$ \\
\hline $\begin{array}{l}\text { Graves'disease } \\
(\mathrm{n}=24)\end{array}$ & 3.89 & 3.44 & 0.006 & 17.98 & 41.79 \\
\hline Painless thyroiditis & $(1.55-5.41)$ & $(1.28-6.98)$ & $(0.001-0.015)$ & $(10-49.09)$ & $(31.15-99.09)$ \\
$(\mathrm{n}=13)$ & 0.46 & 0.93 & 0.007 & 30.5 & 45.55 \\
\hline $\begin{array}{l}\text { Thyrotoxicosis } \\
(\mathrm{n}=37)\end{array}$ & $(0.39-1.58)$ & $(0.72-1.37)$ & $(0.001-0.013)$ & $(14.54-49.09)$ & $(23.85-99.09)$ \\
\hline $\begin{array}{l}\text { Control group } \\
(\mathrm{n}=15)\end{array}$ & 2.93 & 2.81 & 0.006 & 28.43 & 44.54 \\
& $\mathrm{~N} / \mathrm{D}-5.41)$ & $(0.72-6.98)$ & $(0.001-0.02)$ & $(10-49)$ & $(23.85-99.09)$ \\
\hline
\end{tabular}

radioreceptor assay (DYNO test TRAb human; Yamasa Co., Chiba, Japan). The normal range for TRAb was set below 1.0 IU/l. The time delay between the diffusion MR imaging of the thyroid gland and thyroid function tests ranged between $4-6$ days.

\section{Statistical analysis}

The statistical analysis of data was performed with Statistical Package for Social Science software, version 21 (SPSS Inc., Chicago, Ill, USA). Qualitative data were described with counts and percentages. Associations between categorical variables was tested with the chisquared test. For parametric data, continuous variables were presented as means and standard deviations (mean $\pm \mathrm{SD}$ ). The Kolmogorov-Smirnov test was used to test the normality of quantitative data. The ADC values were normally distributed, whereas the Tc-99m uptake levels and TRAb, TSH, FT3, and FT4 levels were non-normal. For parametric data, Student's t tests and one-way analysis of variance (ANOVA) were used. Mann-Whitney U tests and Kruskal-Wallis tests were used to compare non-parametric variables. ROC (Receiver Operating Characteristic) curves were drawn to detect optimal cut-off $A D C$ values for differentiation between patients with thyrotoxicosis and controls. This ADC value, Tc-99m uptake levels, and TRAb levels discriminated between Graves' disease and painless thyroiditis with the best accuracy. $P$ value of $\leq 0.05$ was considered to be statistically significant. The Spearman's coefficient was used to evaluate the degree of correlation between the ADC value of the thyroid gland, Tc-99m uptake levels, and laboratory tests (TRAb, TSH, FT4, and FT3).

\section{Results}

This study included the three following groups: a group of patients with Graves' disease $(\mathrm{n}=24)$ (Figure 1), a group of patients with painless thyroiditis $(n=13)$, and a control 


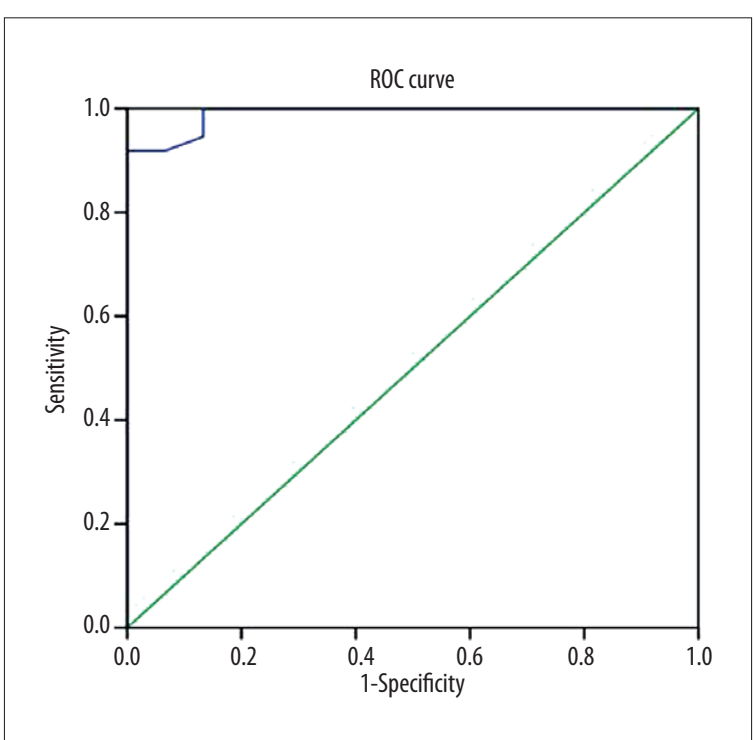

Figure 2. Receiver operating characteristic (ROC) curve. The cut-off $A D C$ value that was used for differentiating thyrotoxicosis from normal thyroid parenchyma was $\geq 1.3 \times 10^{-3} \mathrm{~mm}^{2} / \mathrm{sec}$, with sensitivity of $91.9 \%$, specificity of $100 \%$, accuracy of $94.2 \%$, and area under the curve of 0.99 .

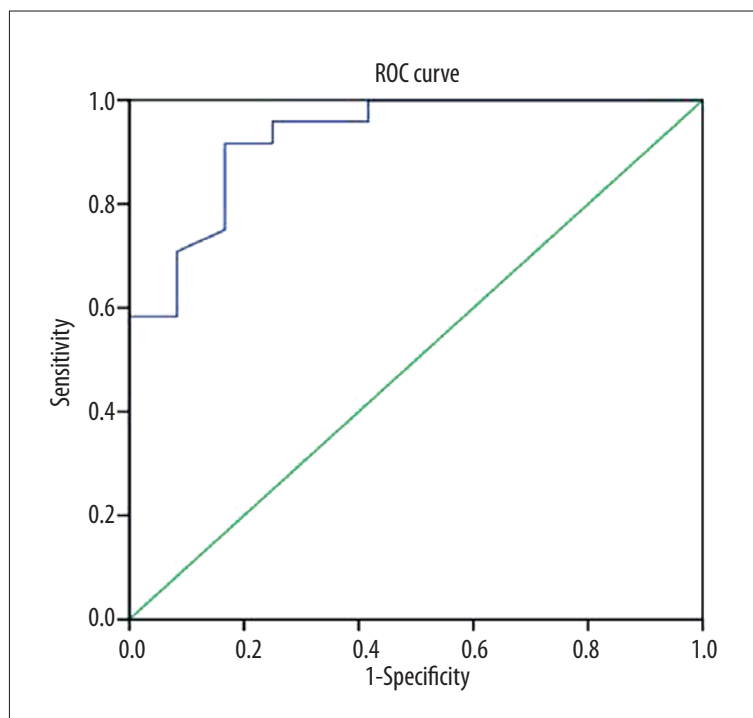

Figure 3. Receiver operating characteristic (ROC) curve. The cut-off $A D C$ value that was used for differentiating patients with Graves' disease from patients with painless thyroiditis was $\geq 1.45 \times 10^{-3} \mathrm{~mm}^{2} / \mathrm{sec}$, with sensitivity of $95.8 \%$, specificity of $61.5 \%$, accuracy of $83.8 \%$, and area under the curve of 0.934 .

group $(\mathrm{n}=15)$. Table 1 shows the ADC values, and Table 2 shows the Tc-99m uptake levels and laboratory tests (TRAb, TSH, FT3, and FT4) of patients and controls.

The mean ADC value of the thyroid gland in patients with thyrotoxicosis was $1.83 \pm 0.38 \times 10^{-3} \mathrm{~mm}^{2} / \mathrm{sec}$, and in the control group it was $1.02 \pm 0.14 \times 10^{-3} \mathrm{~mm}^{2} / \mathrm{sec}$. There was a significant difference in the $\mathrm{ADC}$ value between patients with thyrotoxicosis and controls $(P=0.001)$. The cut-off $\mathrm{ADC}$ value that was used for differentiating patients from controls was $\geq 1.3 \times 10^{-3} \mathrm{~mm}^{2} / \mathrm{sec}$, with area under the curve

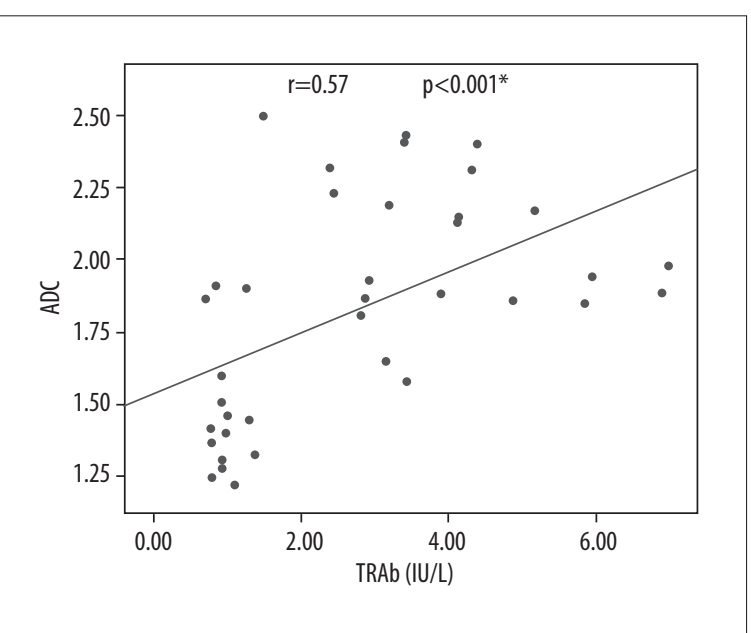

Figure 4. Scatter plot showing a positive correlation between $A D C$ and Tc-99m uptake values. There is a positive correlation between the $A D C$ and $T c-99 \mathrm{~m}$ uptake values $(r=0.74$, $\mathrm{P}=0.001)$.

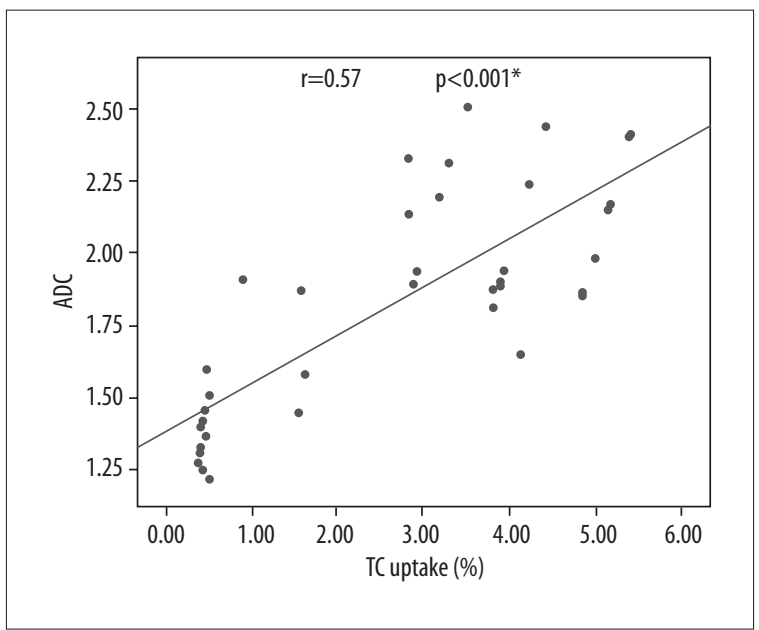

Figure 5. Scatter plot showing correlation between $A D C$ values and TRAb levels. There is a positive correlation between the $A D C$ values and the serum level of TRAb $(r=0.57, P=0.001)$.

of 0.99 , accuracy of $94.2 \%$, sensitivity of $91.9 \%$, specificity of $100 \%$, positive predictive value of $100 \%$, and negative predictive value of $83.3 \%$ (Figure 2). There was a significant difference in serum TSH, FT3, and FT4 levels between both patient groups and the control group $(P=0.001)$.

The mean ADC value in patients with Graves' disease was $2.03 \pm 0.28 \times 10^{-3} \mathrm{~mm}^{2} / \mathrm{sec}$, and in patients with painless thyroiditis it was $1.46 \pm 0.22 \times 10^{-3} \mathrm{~mm}^{2} / \mathrm{sec}$. There was a significant difference in the ADC value between patients with Graves' disease and painless thyroiditis $(P=0.001)$. The cut-off $\mathrm{ADC}$ value of the thyroid gland that was used for differentiating Graves' disease from painless thyroiditis was $\geq 1.45 \times 10^{-3} \mathrm{~mm}^{2} / \mathrm{sec}$, with area under the curve of 0.934 , accuracy of $83.8 \%$, sensitivity of $95.8 \%$, specificity of $61.5 \%$, positive predictive value of $82.1 \%$, and negative predictive value of $88.9 \%$ (Figure 3).

The median Tc-99m uptake values in patients with Graves' disease $(3.89 \%)$ were significantly different $(P=0.001)$ from 
those found in patients with painless thyroiditis $(0.46 \%)$. The cut-off value of Tc-99m uptake that was used to discriminate Graves' disease from painless thyroiditis was $\geq 1.23$, with area under the curve of 0.997 , accuracy of $97.3 \%$, sensitivity of $100 \%$, specificity of $92.3 \%$, positive predictive value of $96 \%$, and negative predictive value of $100 \%$. There was a positive correlation between the $\mathrm{ADC}$ values and Tc-99m uptake values $(r=0.74, P=0.001)$ (Figure 4).

The median serum level of TRAb in patients with Graves' disease $(3.44 \mathrm{IU} / \mathrm{ml})$ was significantly higher $(P=0.001)$ than in patients with painless thyroiditis $(0.93 \mathrm{IU} / \mathrm{ml})$. The serum level of TRAb in patients with thyrotoxicosis showed a positive correlation with ADC values $(r=0.57$, $P=0.001$ ) (Figure 5). The ADC values of the thyroid gland in patients with thyrotoxicosis revealed a positive correlation with serum TSH $(r=0.22, P=0.39)$, and a negative correlation with serum FT3 $(r=-0.28, P=0.22)$ and FT4 $(r=-0.29$, $P=0.29$ ).

\section{Discussion}

The main findings of this study are that the $\mathrm{ADC}$ value of the thyroid gland, Tc-99m thyroid uptake, and serum TRAb levels are significantly different in patients with Graves' disease in comparison to patients with painless thyroiditis. The $\mathrm{ADC}$ value of the thyroid gland is well correlated with Tc-99m thyroid uptake and serum TRAb levels.

In the current study, there was a significant difference $(P=0.001)$ in the ADC value of the thyroid gland parenchyma in patients with thyrotoxicosis and controls. A previous study reported that the mean ADC values of the thyroid gland in healthy volunteers were $2.93 \times 10^{-3} \mathrm{~mm}^{2} / \mathrm{sec}$, $1.97 \times 10^{-3} \mathrm{~mm}^{2} / \mathrm{sec}$, and $1.62 \times 10^{-3} \mathrm{~mm}^{2} / \mathrm{sec}$ at $\mathrm{b}=100$, $b=600$, and $b=1000 \mathrm{sec} / \mathrm{mm}^{2}$, respectively. Also, the optimal threshold values of ADC and TRAb that was used to differentiate patients from controls were $1.837 \times 10^{-3}$ $\mathrm{mm}^{2} / \mathrm{sec}$ and $1.350 \mathrm{IU} / \mathrm{ml}$, respectively [26].

One study reported that the optimal threshold ADC value that was used to differentiate Graves' disease from painless thyroiditis was $1.837 \times 10^{-3} \mathrm{~mm}^{2} / \mathrm{sec}$, with sensitivity, specificity, accuracy, PPV, and NPV of 96.078, 91.892, $95.000,97.059$ and $89.474 \%$, respectively [27]. Another study added that the ADC value was significantly higher in patients with Graves' disease in comparison to patients with painless thyroiditis $(P=0.05)$; the mean ADC values of the thyroid gland in Graves' disease were 3.47, 2.25 and $1.64 \times 10^{-3} \mathrm{~mm}^{2} / \mathrm{sec}$, and in Hashimoto thyroiditis they were $2.53,1.76,1.28 \times 10^{-3} \mathrm{~mm}^{2} / \mathrm{sec}$ for $b$-values of 100,600 , and $1000 \mathrm{sec} / \mathrm{mm}^{2}$, respectively. The ADC values obtained in patients with Graves' disease were higher than those observed in patients with Hashimoto's thyroiditis [26]. Another study reported that the ADC values found in patients with Graves' disease were significantly higher than those observed in patients with subacute thyroiditis and Hashimoto's thyroiditis (sensitivity and specificity of $75 \%$ and $80 \%$, respectively) [24].

In this study, the ADC values that were observed in patients with Graves' disease were significantly higher in comparison to those found in patients with painless thyroiditis. This is attributed to different pathological changes in both diseases. Graves' disease is characterized by diffuse hyperplasia and hypertrophy of follicular cells, preservation of lobular architecture, prominent vascular congestion with tall follicular cells, and papillae that are associated with unrestricted diffusion and higher $\mathrm{ADC}$ values. In contrast, painless thyroiditis is caused by disrupted thyroid follicles which results in a release of preformed thyroid hormones. This process is associated with a diffuse lymphocytic infiltration in the thyroid gland. Hypercellularity in painless thyroiditis leads to numerous structural components and membranes, resulting in greater impedance and restriction of diffusion of water molecules, which leads to lower ADC values [24,26,27].

In this study, the mean Tc-99m uptake values in Graves' disease were significantly different $(P=0.001)$ from those observed in painless thyroiditis. There was a positive correlation between the $\mathrm{ADC}$ values of the thyroid gland and Tc-99m uptake values in all patients $(r=0.74, P=0.001)$. Previous studies reported that thyroid scintigraphy is of a high diagnostic value for differentiating between Graves' disease and painless thyroiditis, and it has been demonstrated to be reliable [11-14]. Thyroid scintigraphy can indicate an enhanced isotope uptake in Graves' disease as well as disruption of thyroid follicles and suppressed uptake in painless thyroiditis.

In the present study, the serum levels of TRAb were significantly higher in Graves' disease than in painless thyroiditis, and both groups showed a positive correlation with the obtained ADC values $(r=0.57, P=0.001)$. The TRAb titer has been demonstrated to be a useful serum marker that can be used to differentiate Graves' disease from painless thyroiditis, although false positives and false negatives can occur; $5-10 \%$ of patients with Graves' disease are negative for TRAb, and some patients with painless thyroiditis have positive TRAb titers [2,3,15-19].

One study reported that the optimal threshold of TRAb that was used to differentiate Graves' disease from painless thyroiditis was $1.350 \mathrm{IU} / \mathrm{ml}$, with sensitivity, specificity, accuracy, $\mathrm{PPV}$, and NPV of $88.235 \%, 75.676 \%, 84.892 \%, 90.909 \%$, and $70.000 \%$ respectively [27].

In this study, there was a positive correlation between ADC values and serum TSH levels, and a negative correlation with serum FT3 and FT4 in patients with thyrotoxicosis. A previous study reported that the ADC value of the thyroid gland in patients with Graves' disease had a positive correlation with TSH $(r=0.87, P=0.001)$, and a negative correlation with serum $\mathrm{T} 4(\mathrm{r}=-0.82, \mathrm{P}=0.001)$ and serum $\mathrm{T} 3$ $(r=-0.71, P=0.001)$ [25]. On the other hand, another study reported that the relationship between the $\mathrm{ADC}$ values of the thyroid gland, serum free T3, and serum free T4 levels was not significant in patients with diffuse thyroid disease [24]. The difference between that study and our study can be attributed to different $b$ values and inclusion of patients with toxic nodular goiter and not with diffuse thyroid disease in [24]. 
There are a few limitations of this study. First, this study was conducted in a small group of patients, with the use of a 1.5-tesla scanner. The application of a 3-tesla scanner could improve the results. Second, as image analysis depends upon ROI selection, application of advanced image processing tools, such as histogram analysis, could increase the diagnostic performance of this technique. Third, this study applied diffusion-weighted MR imaging for assessing thyrotoxicosis. It is recommended that further studies use multi-parametric imaging with diffusion tensor MR imaging, perfusion weighted MR imaging, and MR spectroscopy, which also could improve the results [28-32]. Fourth, there is no standard method for measuring $\mathrm{ADC}$ values and different methods of measuring ADC were not compared. Further evaluations that address these limitations are necessary.

\section{Conclusions}

We concluded that ADC values of the thyroid gland could be used to differentiate Graves' disease from painless thyroiditis in patients with untreated thyrotoxicosis.

\section{Conflict of interest}

The authors declare that they have no conflict of interest.

\section{References:}

1. De Leo S, Lee SY, Braverman LE: Hyperthyroidism. Lancet, 2016; 388: $906-18$

2. Franklyn JA, Boelaert K: Thyrotoxicosis. Lancet, 2012; 379: 1155-66

3. Bartalena L: Diagnosis and management of Graves disease: A global overview. Nat Rev Endocrinol, 2013; 9: 724-34

4. Nishimaki M, Isozaki O, Yoshihara A et al: Clinical characteristics of frequently recurring painless thyroiditis: Contributions of higher thyroid hormone levels, younger onset, male gender, presence of thyroid autoantibody and absence of goiter to repeated recurrence. Endocr J, 2009; 56: 391-97

5. Sipos JA, Kahaly GJ: Imaging of thyrotoxicosis. Am J Med, 2012; 125: S1-2

6. Ide A, Amino N, Yoshioka W et al: Differentiation of postpartum Graves' thyrotoxicosis from postpartum destructive thyrotoxicosis by anti-TSH receptor antibodies and thyroid blood flow. Thyroid, 2014; 24: 1027-31

7. Kamijo K: Study on cutoff value setting for differential diagnosis between Graves' disease and painless thyroiditis using the TRAb (Elecsys TRAb) measurement via the fully automated electrochemiluminescence immunoassay system. Endocr J, 2010; 57: 895-902

8. Nishimaki M, Isozaki O, Yoshihara A et al: Clinical characteristics of frequently recurring painless thyroiditis: Contributions of higher thyroid hormone levels, younger onset, male gender, presence of thyroid autoantibody and absence of goiter to repeated recurrence. Endocr J, 2009; 56: 391-97

9. Yoshimura Noh J, Momotani N et al: Ratio of serum free triiodothyronine to free thyroxine in Graves' hyperthyroidism and thyrotoxicosis caused by painless thyroiditis. Endocr J, 2005; 52: $537-42$

10. Alzahrani AS, Ceresini G, Aldasouqi SA: Role of ultrasonography in the differential diagnosis of thyrotoxicosis: A noninvasive, costeffective, and widely available but underutilized diagnostic tool. Endocr Pract, 2012; 18: 567-78

11. Banaka I, Kaltsas G, Antoniou S et al: Prognostic value of vascularity index for the diagnosis of autoimmune thyroid disease. JBR-BTR 2011; 94: 185-90

12. Hiraiwa $\mathrm{T}$, Tsujimoto $\mathrm{N}$, Tanimoto $\mathrm{K}$ et al: Use of color Doppler ultrasonography to measure thyroid blood flow and differentiate Graves' disease from painless thyroiditis. Eur Thyroid J, 2013; 2: $120-26$

13. Donkol RH, Nada AM, Boughattas S: Role of color Doppler in differentiation of Graves' disease and thyroiditis in thyrotoxicosis. World J Radiol, 2013; 5: 178-83

14. Zhao X, Chen L, Li L et al: Peak systolic velocity of superior thyroid artery for the differential diagnosis of thyrotoxicosis. PLoS One, 2012; 7: e50051

15. Zuhur SS, Ozel A, Kuzu I et al: The diagnostic utility of color Doppler ultrasonography, TC-99M pertechnetate uptake and TSH receptor antibody for differential diagnosis between Graves' disease and silen thyroiditis: A comparative study. Endocr Pract, 2014; 20(4): 310-19
16. Uchida T, Suzuki R, Kasai T et al: Cutoff value of thyroid uptake of (99m)Tc-pertechnetate to discriminate between Graves' disease and painless thyroiditis: A single center retrospective study. Endocr $\mathrm{J}$, 2016; 63: $143-49$

17. Schwartz F, Bergmann N, Zerahn B, Faber J: Incidence rate of symptomatic painless thyroiditis presenting with thyrotoxicosis in Denmark as evaluated by consecutive thyroid scintigraphies. Scand J Clin Lab Invest, 2013; 73: 240-44

18. Abdel Razek AA, Khairy M, Nada N: Diffusion-weighted MR imaging in thymic epithelial tumors: Correlation with world health organization classification and clinical staging. Radiology, 2014; 273: 268-75

19. Razek AA: Diffusion-weighted magnetic resonance imaging of head and neck. J Comput Assist Tomogr, 2010; 34: 808-15

20. Abdel Razek AA, Gaballa G, Denewer A, Tawakol I: Diffusion weighted MR imaging of the breast. Acad Radiol, 2010; 17: 382-86

21. Wu LM, Chen XX, Li YL et al: On the utility of quantitative diffusionweighted MR imaging as a tool in differentiation between malignant and benign thyroid nodules. Acad Radiol, 2014; 21: 355-63

22. Erdem G, Erdem T, Karakas H et al: Diffusion-weighted images differentiate benign from malignant thyroid nodules. J Magn Reson Imaging, 2010; 31: 31: 94-100

23. Abdel Razek A, Sadek A et al: Role of apparent diffusion coefficient value in differentiation between malignant and benign solitary thyroid nodule. Am J Neuroradiol, 2008; 29: 563-68

24. Tezuka M, Murata Y, Ishida R et al: MR imaging of the thyroid: Correlation between apparent diffusion coefficient and thyroid gland scintigraphy. J Magn Reson Imag, 2003; 17: 163-69

25. Abdel Razek AA, Sadek AG, Gaballa G: Diffusion-weighed MR of the thyroid gland in Graves' disease: Assessment of disease activity and prediction of outcome. Acad Radiol, 2010; 17: 779-83

26. Ozturk T, Bozgeyik Z, Ozturk F et al: The role of diffusion weighted MR imaging for differentiation between Graves' disease and Hashimoto thyroiditis. Eur Rev Med Pharmacol Sci, 2015; 19: 2798803

27. Meng Z, Zhang G, Sun $\mathrm{H}$ et al: Differentiation between Graves' disease and painless thyroiditis by diffusion-weighted imaging, thyroid iodine uptake, thyroid scintigraphy and serum parameters. Exp Ther Med, 2015; 9: 2165-72

28. Abdel Razek AA, Gaballa G: Role of perfusion magnetic resonance imaging in cervical lymphadenopathy. J Comput Assist Tomogr 2011; 35: 21-25

29. Abdel Razek AA, Gaballa G, Ashamalla G et al: Dynamic susceptibility contrast perfusion-weighted MR imaging and diffusion-weighted MR imaging in differentiating recurrent head and neck cancer from postradiation changes. J Comput Assist Tomogr, 2015; 39: 849-54

30. Abdel Razek AA, Poptani H: MR spectroscopy of head and neck cancer. Eur J Radiol, 2013; 82: 982-89

31. Abdel Razek AA, Soliman NY, Elkhamary S et al: Role of diffusion weighted MR imaging in cervical lymphadenopathy. Eur Radiol 2006; 16: 1468-77

32. Razek AA, Nada N: Correlation of choline/creatine and apparent diffusion coefficient values with the prognostic parameters of head and neck squamous cell carcinoma. NMR Biomed, 2016; 29: 483-89 